\title{
Herpes zoster in people who are immunocompromised: what are the options for prevention?
}

Charlotte Warren-Gash and Judith Breuer

\begin{abstract}
"Of all the classical precipitants of zoster, two-leukaemia and x-rays-are at present the most important", said R Edgar Hope-Simpson in 1965.1 The link between compromised immunity and herpes zoster has been recognised for more than half a century. Individuals who are severely immunocompromised due to immunosuppressive conditions or therapies have herpes zoster more frequently and severely than the general immunocompetent population. Herpes zoster incidence per 1000 person-years at risk is reported to be 43.03 in adults with bone-marrow or stem-cell transplants, 17.04 in adults with solid organ transplants, and 17.43 in adults with HIV, compared with 4.82 in the general population.2Complications of herpes zoster are also roughly three times higher in people with HIV than in an age-matched general population.3 Furthermore, severe immunocompromise is a contraindication to receiving live attenuated varicella zoster virus vaccine because of the potential risk of the vaccine virus replicating to cause disease. Preventing herpes zoster and its complications in individuals who are severely immunocompromised, therefore, remains an important public health goal.
\end{abstract}

In The Lancet Infectious Diseases, Kathleen Mullane and colleagues4 report promising efficacy and safety of a $y$ irradiation-inactivated varicella zoster virus vaccine (vOka strain) in patients with solid tumour malignancies receiving chemotherapy. In the randomised, doubleblind, placebo-controlled, phase 3 trial done across 40 countries, the primary endpointherpes zoster incidence-was markedly reduced in patients with solid tumour malignancies receiving vaccine compared with those receiving placebo (22 vs 61 cases; vaccine efficacy $63.6 \%, 97 \cdot 5 \% \mathrm{Cl} 36 \cdot 4$ to $79 \cdot 1)$. These results came from 2678 patients with solid tumour malignancies on chemotherapy followed up for a mean of 2.45 years (SD 1.52) who each received at least one dose of the vaccine (the modified intention-to-treat population). The vaccine did not, however, reduce herpes zoster incidence in 2552 patients with haematological malignancies who received at least one vaccine dose (vaccine efficacy $16 \cdot 8 \%, 97 \cdot 5 \% \mathrm{Cl}-17 \cdot 8$ to $41 \cdot 3)$.

The vaccine was well tolerated in patients with solid tumour malignancies receiving chemotherapy, with no differences between groups in frequencies of serious adverse events or vaccine-related serious adverse events up to 28 days after the fourth dose. Vaccinerelated adverse events, which were typically mild injection-site reactions, were, however, more common in those receiving the $y$ irradiation-inactivated vaccine than in those receiving placebo $(36 \cdot 2 \%$ vs $14 \cdot 1 \%)$. A similar safety profile was seen in patients with haematological malignancies.

Non-live vaccines are likely to hold the key to preventing herpes zoster in individuals who are immunocompromised. However, questions remain about which vaccine to use for which patient group. The study by Mullane and colleagues highlights the heterogeneity of vaccine responses between patients with different immunocompromising conditions. It also raises an important issue about the validity of immunogenicity endpoints in vaccine trials. Although individuals with haematological malignancies in this trial appeared to mount an effective immune response to the $\mathrm{y}$-irradiated vaccine, this response did not translate into clinical efficacy. 4 
Another non-live herpes zoster vaccine (Shingrix; GlaxoSmithKline, King of Prussia, PA, USA) is also undergoing clinical trials in patients who are immunocompromised. Licensed to prevent herpes zoster and post-herpetic neuralgia in adults aged 50 years and older, this adjuvanted recombinant vaccine is highly efficacious in older adults who are immunocompetent (vaccine efficacy of around $90 \%$ in all age groups from 50 years). 5 Its safety and immunogenicity have been established in phase $1 / 2$ trials in recipients of autologous haemopoietic stem-cell transplants 6 and people with HIV, 3 as well as in phase 3 trials in patients who have had renal transplantation.7 Phase 3 trial data published alongside the Article of Mullane and colleagues showed safety and immunogenicity of Shingrix in patients with haematological malignancies receiving immunosuppressive therapies.8 Early trial results suggest that efficacy of this vaccine against incident herpes zoster is $87 \%$ in patients with haematological malignancies, $968 \%$ in individuals with autologous haemopoietic stem-cell transplants, 10 and $68 \%$ in patients who have had a renal transplant (NCT01610414). The efficacy of the $y$ irradiation-inactivated vaccine in recipients of autologous haemopoietic stem-cell transplants was $64 \%$ in a phase 3 trial.11

The duration of protection conferred by non-live vaccines in individuals who are severely immuno-compromised remains unclear. Despite administration of four vaccine doses each a month apart in the study by Mullane and colleagues, response to the $y$ irradiation-inactivated vaccine waned markedly over time (vaccine efficacy of around $80 \%$ for months $0-12$ vs $44 \%$ beyond 1 year). Duration of immunity has not yet been reported for the attenuated recombinant vaccine in individuals who are immunocompromised, although vaccine efficacy remains higher than $88 \%$ against incident herpes zoster at 4 years in older adults who are immunocompetent.5

In summary, non-live vaccines offer new hope for preventing herpes zoster and its costly complications in individuals who are immunocompromised. Although implementation plans have yet to be finalised, some countries such as the UK are likely to recommend the recombinant herpes zoster vaccine for patients who are immunocompromised and aged 50 years or older in the coming months. Large studies with clinically meaningful endpoints will provide further insights into the relative efficacies, duration of protection, long-term safety, and cost-effectiveness of non-live zoster vaccines for different immunocompromised groups.

We declare no competing interests. CWG is supported by a Wellcome Intermediate Clinical Fellowship (201440_Z_16_Z). JB receives funding from the UCL/UCLH NIHR Biomedical Research Centre. The funding sources had no role in the writing of this report or in the decision to submit for publication.

\section{References}

1 RE Hope-Simpson The nature of herpes zoster: a long-term study and a new hypothesis Proc R Soc Med, 58 (1965), pp. 9-20

2 SY Chen, JA Suaya, Q Li, et al. Incidence of herpes zoster in patients with altered immune function Infection, 42 (2014), pp. 325-334 
3 EM Berkowitz, G Moyle, HJ Stellbrink, et al. Safety and immunogenicity of an adjuvanted herpes zoster subunit candidate vaccine in HIV-infected adults: a phase 1/2a randomized, placebo-controlled study J Infect Dis, 211 (2015), pp. 1279-1287

4 KM Mullane, VA Morrison, LH Camacho, et al. Safety and efficacy of inactivated varicella zoster virus vaccine in immunocompromised patients with malignancies: a two-arm, randomised, double-blind, phase 3 trial Lancet Infect Dis (2019)

5 AL Cunningham, $\mathrm{H}$ Lal, M Kovac, et al. Efficacy of the herpes zoster subunit vaccine in adults 70 years of age or older N Engl J Med, 375 (2016), pp. 1019-1032

6 EA Stadtmauer, KM Sullivan, FM Marty, et al. A phase 1/2 study of an adjuvanted varicella-zoster virus subunit vaccine in autologous hematopoietic cell transplant recipients Blood, 124 (2014), pp. 2921-2929

7 P Vink, JM Ramon Torrell, A Sanchez Fructuoso, et al. Immunogenicity and safety of the adjuvanted recombinant zoster vaccine in chronically immunosuppressed adults following renal transplant: a phase III, randomized clinical trial Clin Infect Dis (2019)

8 AF Dagnew, O Ilhan, W-S Lee, et al. Immunogenicity and safety of the adjuvanted recombinant zoster vaccine in adults with haematological malignancies: a phase 3 , randomised, clinical trial and post-hoc efficacy analysis Lancet Infect Dis (2019)

9 AF Dagnew, O Ilhan, W-S Lee, et al. Immunogenicity, safety and post-hoc efficacy assessment of the adjuvanted recombinant zoster vaccine in adults with hematologic malignancies: a phase 3, randomized clinical trial IDWeek, San Francisco, CA (Oct 4, 2018), p. 149

10 de la Serna J, Campora L, Chandrasekar P, et al. Efficacy and safety of an adjuvanted herpes zoster subunit vaccine in autologous hematopoietic stem cell transplant recipients 18 years of age or older: first results of the phase 3 randomized, placebo-controlled ZOE-HSCT clinical trial. BMT Tandem meetings; Salt Lake City, UT; Feb 25, 2018. LBA2.

11 DJ Winston, KM Mullane, OA Cornely, et al. Inactivated varicella zoster vaccine in autologous haemopoietic stem-cell transplant recipients: an international, multicentre, randomised, double-blind, placebo-controlled trial Lancet, 391 (2018), pp. 2116-2127 